\title{
ON A RIVER BARGE TYPE EUROPE B2 1740T OPERATION LIMITS EVALUATION IN IRREGULAR WAVES
}

\author{
Elisabeta Burlacu \\ "Dunarea de Jos" University of Galati, \\ Faculty of Naval Architecture, Galati, \\ 47 Domneasca Street, 800008, Romania, \\ E-mail: elisabeta.burlacu@ugal.ro
}

\author{
Leonard Domnișoru \\ "Dunarea de Jos" University of Galati, \\ Faculty of Naval Architecture, Galati, \\ 47 Domneasca Street, 800008, Romania, \\ E-mail: leonard.domnisoru@ugal.ro
}

\begin{abstract}
The barge type Europe B2 1740 T is designed for operation on river routes. According to the cargo loading cases, on Danube River the significant wave height limits for operation in safety conditions have to be evaluated using the dynamic criteria in irregular waves. The study covers three main loading cases, using an eigen program package DYN-OSC for dynamic analysis. A full range of wave heading angles and three speeds are considered. The dynamic criteria are formulated for heave, roll and pitch motions plus accelerations, and results the barge reference operation limits.
\end{abstract}

Keywords: Europe B2 1740T barge, dynamic analysis, irregular waves.

\section{INTRODUCTION}

The operation limits in irregular waves navigation conditions, for each specific loading case of the Europe B2 1740T barge [4], have to be assessed by dynamic criteria [1,2].

For this study we use the eigen program package DYN-OSC [5], developed by a linear hydrodynamic and Lewis models [1], with ITTC wave power density spectrum (Fig.2).

The dynamic criteria are formulated in terms of most probable admissible amplitudes, $R M S$ values, on motions and accelerations for $\zeta$ heave, $\varphi$ roll and $\theta$ pitch [5].

$$
\begin{aligned}
& R M S_{\text {max } \beta}=H_{\beta}-f_{s}-T_{\beta} \geq R M S ; \beta=a, m, f \\
& R M S a c_{\zeta \max }=0.1 g \geq R M S_{a c \zeta} ; f_{s}=0.3 m \\
& R M S_{z}=R M S_{\zeta}+\left(L / 2+\delta \cdot x_{F}\right) \cdot R M S_{\theta}+ \\
& +B / 2 \cdot R M S_{\varphi}+H_{s} / 4 ; \delta \in\{+1,0,-1\} \\
& R M S_{\theta \max }=2 \mathrm{deg} \geq R M S_{\theta} \\
& R M S a c_{\theta \max }=0.1 g /(L / 2) \geq R M S_{a c \theta} \\
& R M S_{\varphi \max }=4 \mathrm{deg} \geq R M S_{\varphi} \\
& R M S a c_{\varphi \max }=0.1 g /(B / 2) \geq R M S_{a c \varphi}
\end{aligned}
$$

where: $a, m, f$ are the aft, mid and fore reference positions; $L, B, T$ are the barge length, breadth and draught; $x_{F}$ is the water plane centre longitudinal position [3].

Table 1 presents the Europe B2 1740T barge main characteristics [4], with offset lines in Fig. 1 and dynamic criteria in Table 2.

Table 1. Barge main characteristics [4]

\begin{tabular}{|c|c|c|c|c|}
\hline$L[\mathrm{~m}]$ & $B[\mathrm{~m}]$ & $H_{a}[\mathrm{~m}]$ & $H_{m}[\mathrm{~m}]$ & $H_{f}[\mathrm{~m}]$ \\
\hline 76.5 & 10.96 & 3.20 & 3.20 & 3.90 \\
\hline$H_{s \text { max }}[\mathrm{m}]$ & $\mu[\mathrm{deg}]$ & $v[\mathrm{~km} / \mathrm{h}]$ & $N s$ & $N_{P}$ \\
\hline $0-2(0.25)$ & $0-360(5)$ & $0,5,10$ & 405 & 21992 \\
\hline Case & $\Delta[\mathrm{t}]$ & $T_{m}[\mathrm{~m}]$ & $x_{F}[\mathrm{~m}]$ & $z_{G}[\mathrm{~m}]$ \\
\hline Barge_1 & 405.95 & 0.55 & -3.586 & 0.810 \\
\hline Barge_2 & 1540.95 & 2.00 & -1.616 & 1.134 \\
\hline Barge_3 & 2108.40 & 2.70 & -0.885 & 1.448 \\
\hline$G M_{T 0}[\mathrm{~m}]:$ & 17.682 & 5.011 & 3.765 & $g\left[\mathrm{~m} / \mathrm{s}^{2}\right]$ \\
\hline$J_{x}\left[\mathrm{tm}^{2}\right]:$ & 4152.36 & 16085.6 & 22578.9 & 9.81 \\
\hline
\end{tabular}

Table 2. Dynamic criteria for barge analysis

\begin{tabular}{|c|c|c|c|c|c|}
\hline Case & $R M S_{z a}$ & $R M S_{z m}$ & $R M S_{z f}$ & $R M S_{\theta}$ & $R M S_{\varphi}$ \\
\hline Barge_1 & 2.35 & 2.35 & 3.05 & & \\
\hline Barge_2 & 0.90 & 0.90 & 1.60 & 0.0349 & 0.0698 \\
\hline Barge_3 & 0.20 & 0.20 & 0.90 & & \\
\hline$R M S_{a c \zeta}$ & 0.9810 & $R M S_{a c \theta}$ & 0.0256 & $R M S_{a c \phi}$ & 0.1790 \\
\hline
\end{tabular}




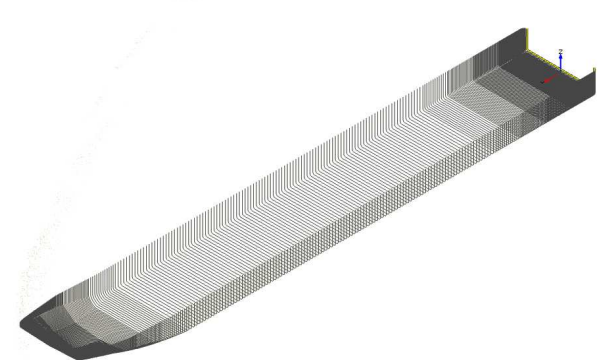

Fig.1 Barge Europe B2 1740T 3D lines [4]

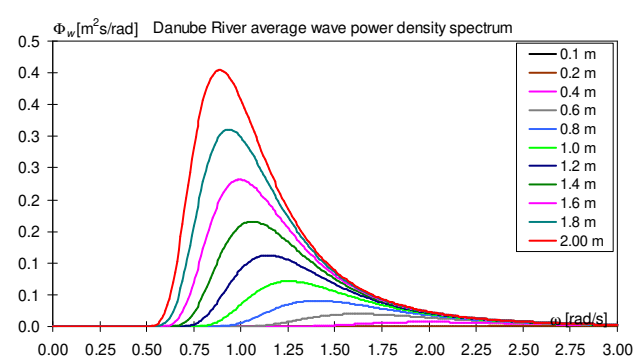

Fig.2 The ITTC wave spectra for $H_{s} \leq 2 \mathrm{~m}$ [1]

Table 3 presents the natural oscillation circular frequencies and Fig. 3 presents the transversal stability diagrams for the three loading cases. Up to $10 \mathrm{deg}$. the transversal stability diagrams are linear, so that a nonlinear roll model is not required.

Table 3. The natural oscillation frequencies for barge Europe B2 1740T, three cargo cases

\begin{tabular}{|l|c|c|c|}
\hline Case & $\omega_{\zeta}[\mathrm{rad} / \mathrm{s}]$ & $\omega_{\theta}[\mathrm{rad} / \mathrm{s}]$ & $\omega_{\varphi}[\mathrm{rad} / \mathrm{s}]$ \\
\hline Barge_1 & 1.603 & 1.507 & 3.999 \\
\hline Barge_2 & 1.365 & 1.341 & 1.176 \\
\hline Barge_3 & 1.251 & 1.244 & 1.433 \\
\hline
\end{tabular}

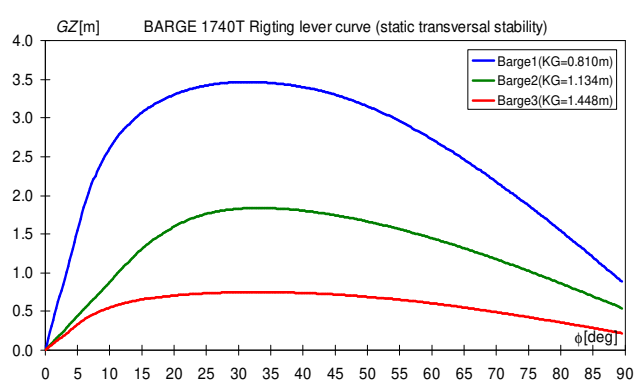

Fig.3 The transversal stability diagrams for barge Europe B2 1740T, three cargo cases

\section{THE DYNAMIC ANALYSIS FOR LIGHT DISPLACEMENT CASE}

In the case of light displacement, Barge_1 (Table 1) the following dynamic results are obtained:

-Figs.3.a-c the response amplitude operators, $v=10 \mathrm{~km} / \mathrm{h}\left(F_{n}=0.102\right)$, for the main motions heave, roll and pitch, for case 1 ;

-Figs.4.a-h the maximum most probable amplitudes, RMS values, compared to the dynamic criteria (Table 2), for case 1 ;

-Figs.5.a,b the operation limits by dynamic criteria, in terms of limit significant wave height $H_{s}$ and Beaufort sea state, speeds $v=0$, $5,10 \mathrm{~km} / \mathrm{h}$ and $\mu=0-360 \mathrm{deg}$., for case 1 .



Fig.3.a Heave $R A O, v=10 \mathrm{~km} / \mathrm{h}$, case 1

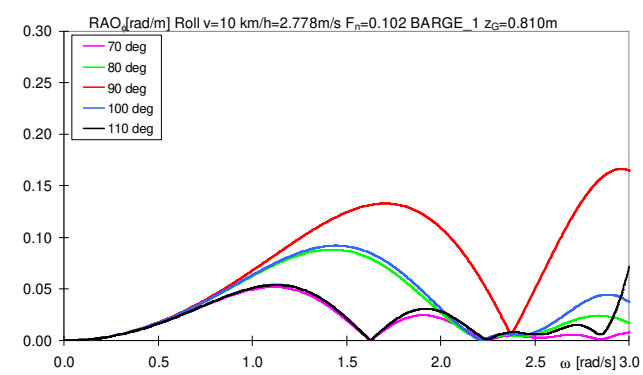

Fig.3.b Roll $R A O, v=10 \mathrm{~km} / \mathrm{h}$, case 1

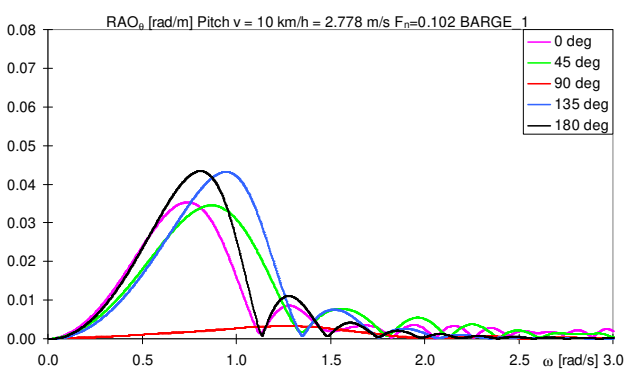

Fig.3.c Pitch $R A O, v=10 \mathrm{~km} / \mathrm{h}$, case 1 


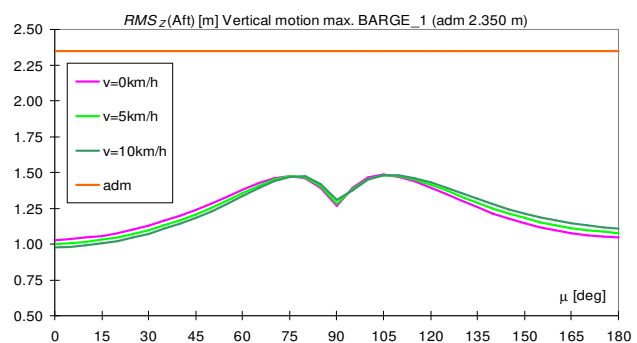

Fig.4.a Vertical $R M S[\mathrm{~m}]$ aft max., case 1

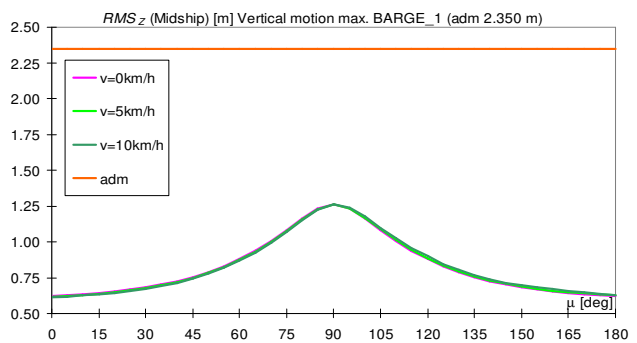

Fig.4.b Vertical $R M S[\mathrm{~m}]$ mid max., case 1

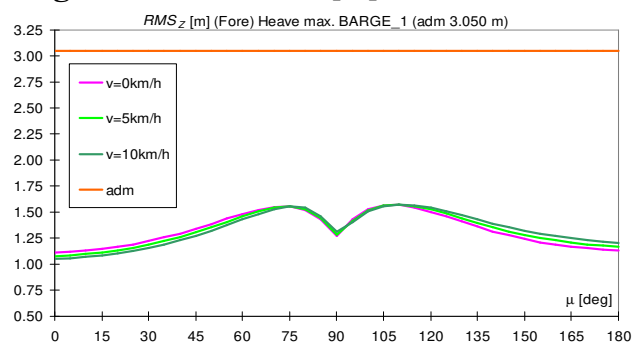

Fig.4.c Vertical RMS [m] fore max., case 1

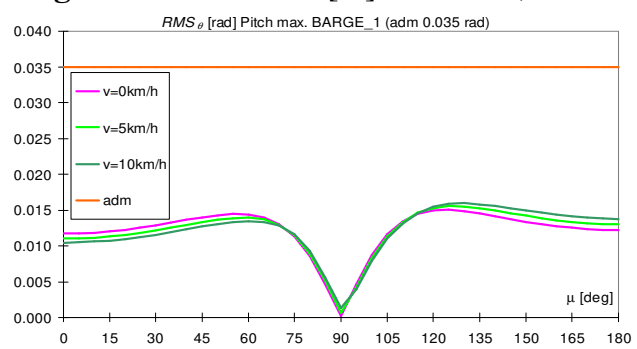

Fig.4.d Pitch $R M S$ [rad] max., case 1

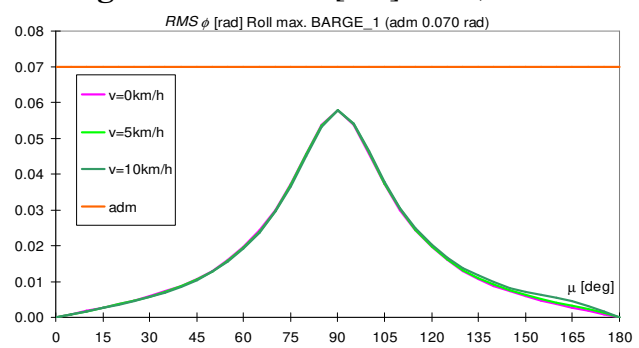

Fig.4.e Roll RMS [rad] max., case 1

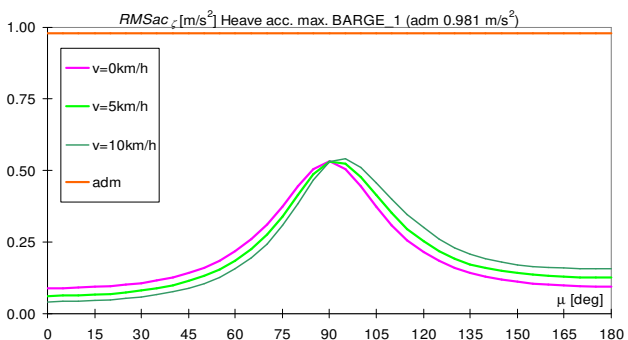

Fig.4.f Vertical acc. $R M S\left[\mathrm{~m} / \mathrm{s}^{2}\right]$ max., case 1

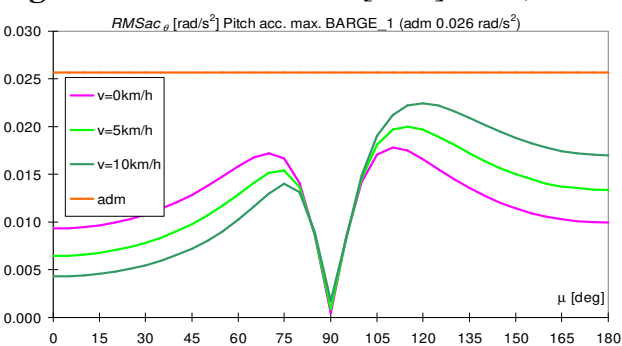

Fig.4.g Pitch acc. $R M S\left[\mathrm{rad} / \mathrm{s}^{2}\right]$ max., case 1

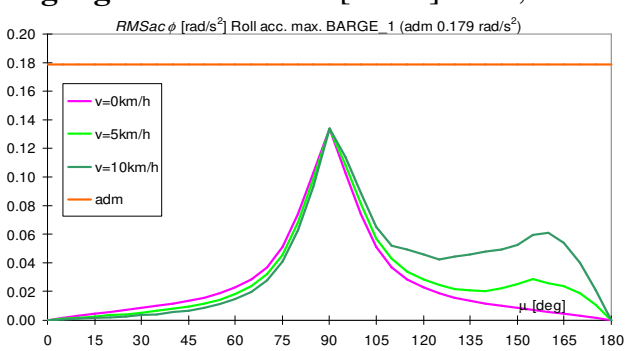

Fig.4.h Roll acc. $R M S\left[\mathrm{rad} / \mathrm{s}^{2}\right]$ max., case 1

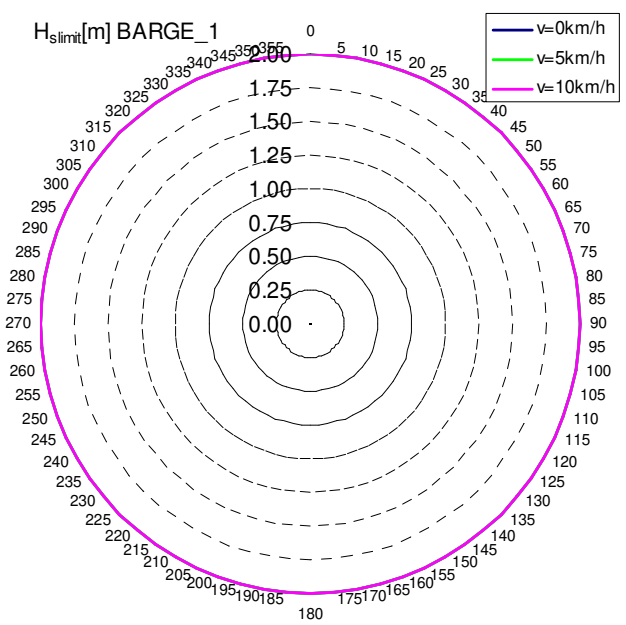

Fig.5.a The operation limits $H_{s}[\mathrm{~m}]$, dynamic criteria, case 1 


\section{THE DYNAMIC ANALYSIS FOR COMMON CARGO LOAD CASE}

In the case of common cargo load case, Barge_2 (Table 1) the following dynamic results are obtained:

-Figs.6.a-c the response amplitude operators, $v=10 \mathrm{~km} / \mathrm{h}\left(F_{n}=0.102\right)$, for the main motions heave, roll and pitch, for case 2 ;

-Figs.7.a-h the maximum most probable amplitudes, $R M S$ values, compared to the dynamic criteria (Table 2), for case 2;

-Figs.8.a,b the operation limits by dynamic criteria, in terms of limit significant wave height $H_{s}$ and Beaufort sea state, speeds $v=0$, $5,10 \mathrm{~km} / \mathrm{h}$ and $\mu=0-360 \mathrm{deg}$., for case 2 .

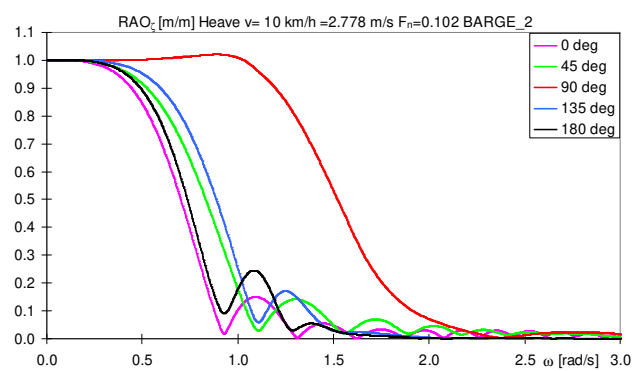

Fig.6.a Heave $R A O, v=10 \mathrm{~km} / \mathrm{h}$, case 2

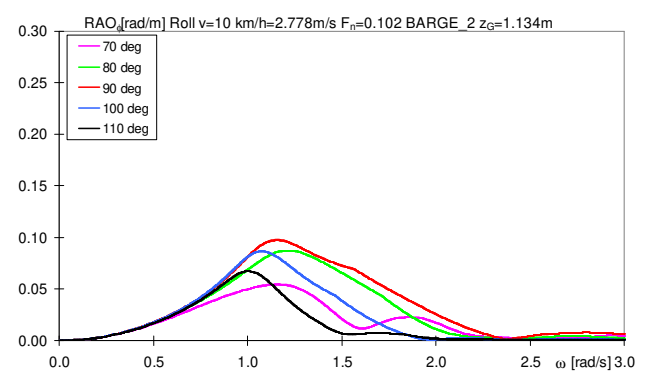

Fig.6.b Roll $R A O, v=10 \mathrm{~km} / \mathrm{h}$, case 2

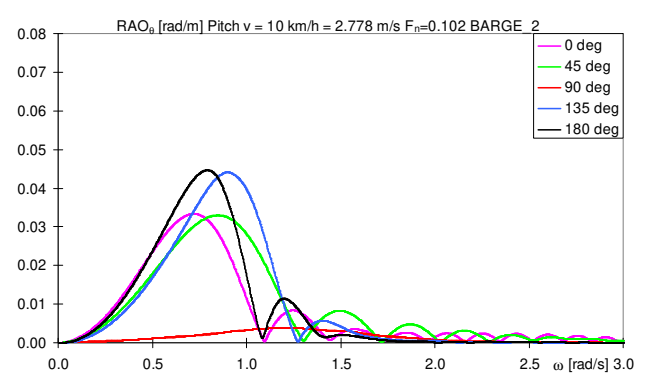

Fig.6.c Pitch $R A O, v=10 \mathrm{~km} / \mathrm{h}$, case 2

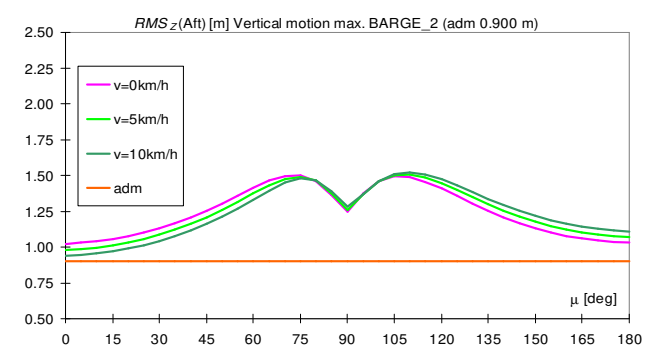

Fig.7.a Vertical $R M S[\mathrm{~m}]$ aft max., case 2

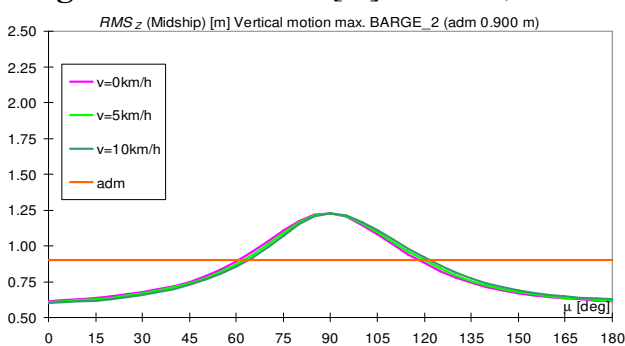

Fig.7.b Vertical RMS [m] mid max., case 2

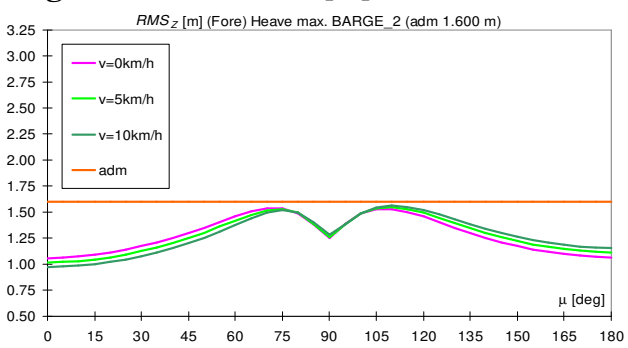

Fig.7.c Vertical RMS [m] fore max., case 2

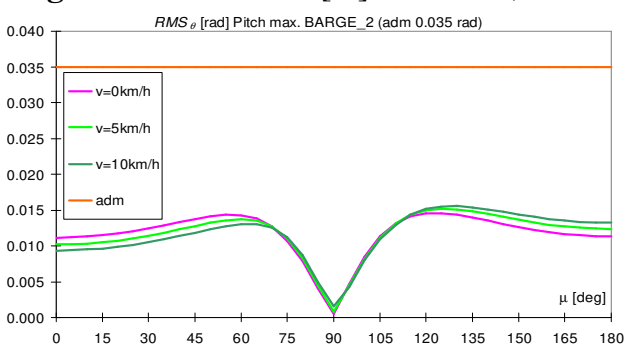

Fig.7.d Pitch $R M S$ [rad] max., case 2

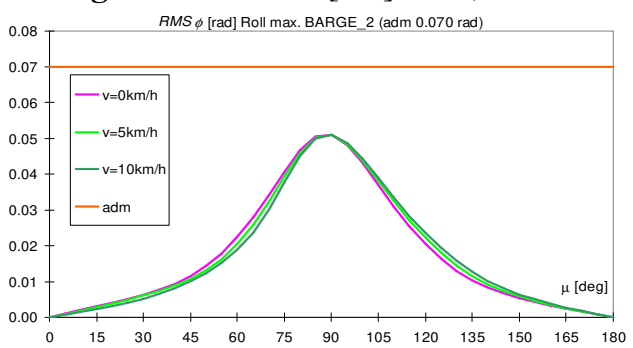

Fig.7.e Roll RMS [rad] max., case 2 


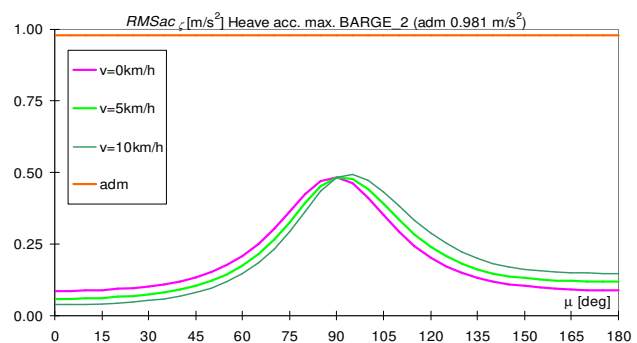

Fig.7.f Vertical acc. $R M S\left[\mathrm{~m} / \mathrm{s}^{2}\right]$ max., case 2

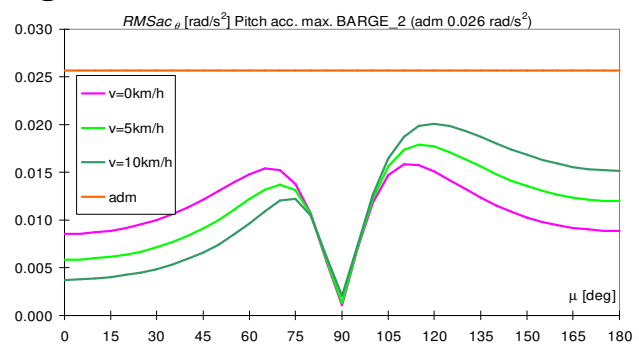

Fig.7.g Pitch acc. $R M S\left[\mathrm{rad} / \mathrm{s}^{2}\right]$ max., case 2

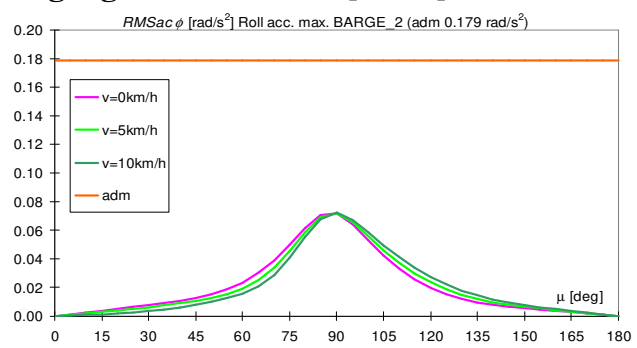

Fig.7.h Roll acc. $R M S\left[\mathrm{rad} / \mathrm{s}^{2}\right]$ max., case 2

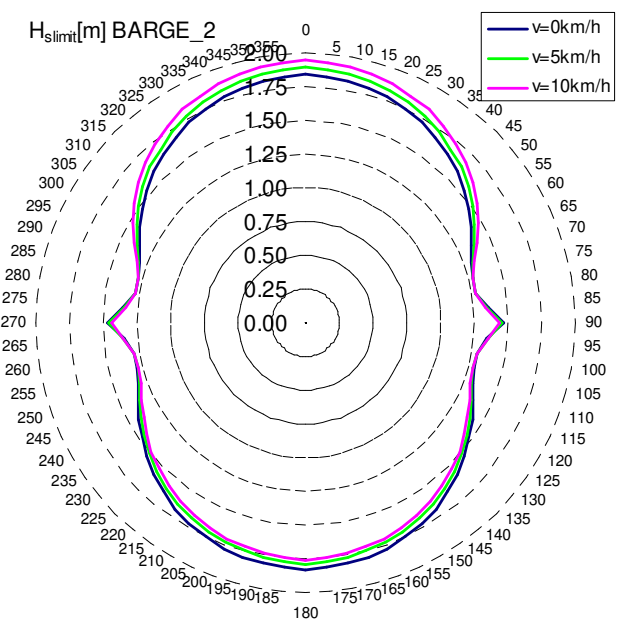

Fig.8.a The operation limits $H_{s}[\mathrm{~m}]$, dynamic criteria, case 2
4. THE DYNAMIC ANALYSIS FOR FULL CARGO LOAD CASE

In the case of full cargo load case, Barge_3 (Table 1) the following dynamic results are obtained:

-Figs.9.a-c the response amplitude operators, $v=10 \mathrm{~km} / \mathrm{h}\left(F_{n}=0.102\right)$, for the main motions heave, roll and pitch, for case 3;

-Figs.10.a-h the maximum most probable amplitudes, $R M S$ values, compared to the dynamic criteria (Table 2), for case 3;

-Figs.11.a,b the operation limits by dynamic criteria, in terms of limit significant wave height $H_{s}$ and Beaufort sea state, speeds $v=0$, $5,10 \mathrm{~km} / \mathrm{h}$ and $\mu=0-360 \mathrm{deg}$., for case 3 .

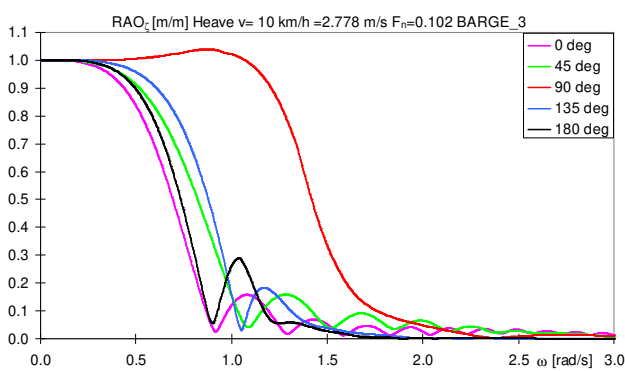

Fig.9.a Heave $R A O, v=10 \mathrm{~km} / \mathrm{h}$, case 3

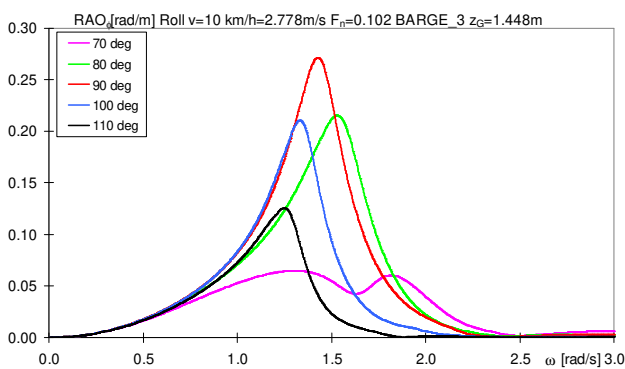

Fig.9.b Roll $R A O, v=10 \mathrm{~km} / \mathrm{h}$, case 3

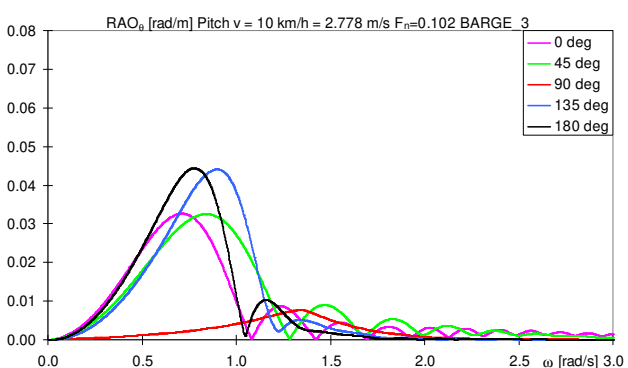

Fig.9.c Pitch $R A O, v=10 \mathrm{~km} / \mathrm{h}$, case 3 


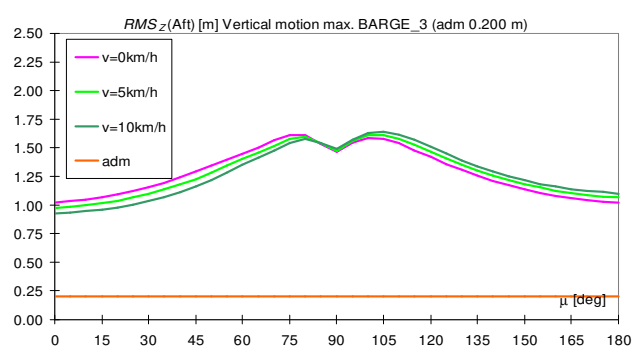

Fig.10.a Vertical $R M S[\mathrm{~m}]$ aft max., case 3

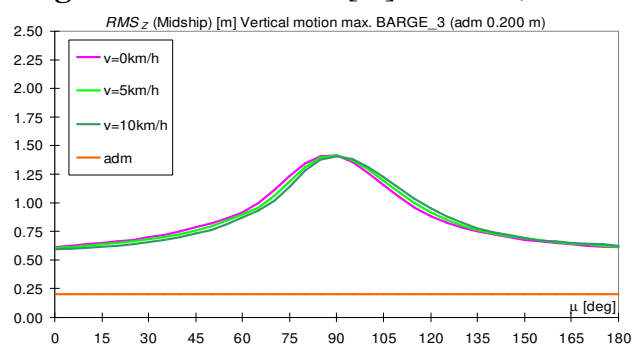

Fig.10.b Vertical $R M S[\mathrm{~m}]$ mid max., case 3

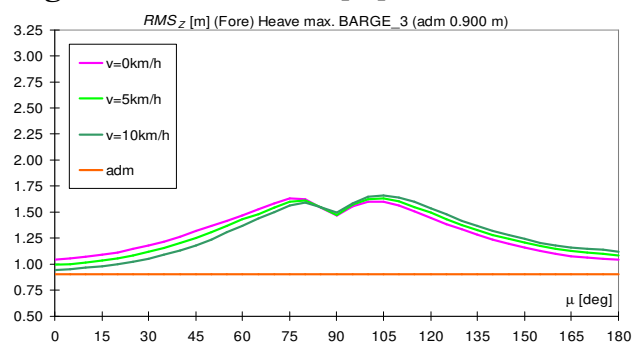

Fig.10.c Vertical RMS [m] fore max., case 3

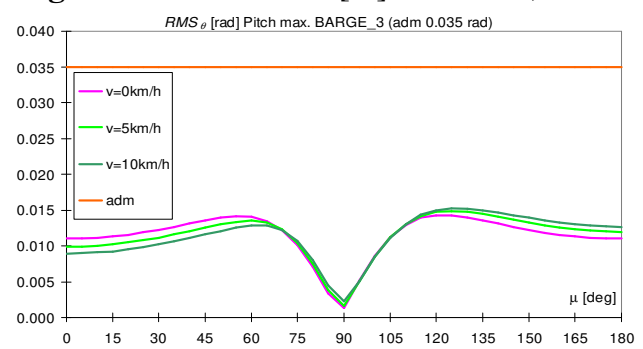

Fig.10.d Pitch $R M S$ [rad] max., case 3

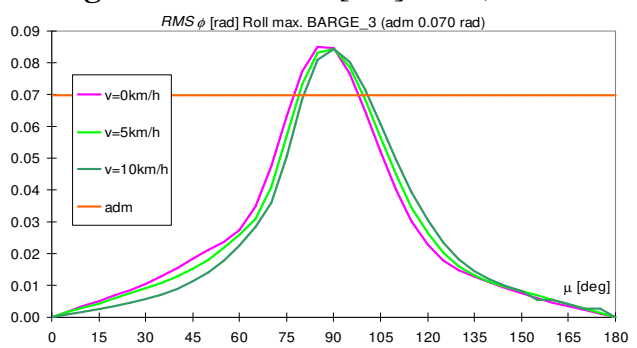

Fig.10.e Roll RMS [rad] max., case 3

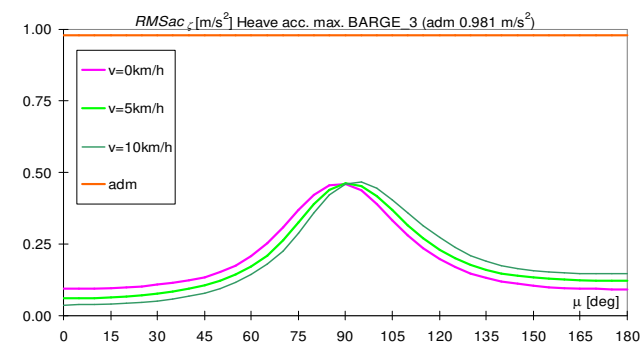

Fig.10.f Vertical acc.RMS [m/ $\left.\mathrm{s}^{2}\right]$ max.,case 3

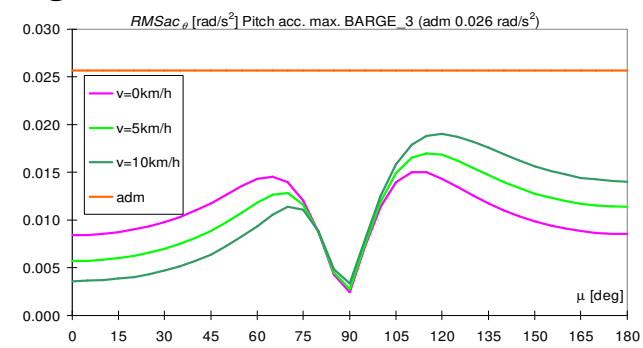

Fig.10.g Pitch acc. $R M S\left[\mathrm{rad} / \mathrm{s}^{2}\right]$ max., case 3

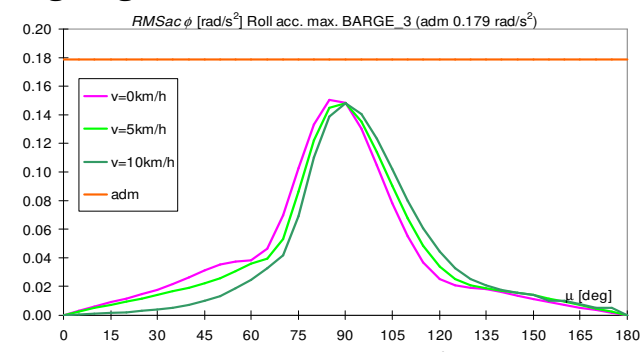

Fig.10.h Roll acc. $R M S\left[\mathrm{rad} / \mathrm{s}^{2}\right]$ max., case 3

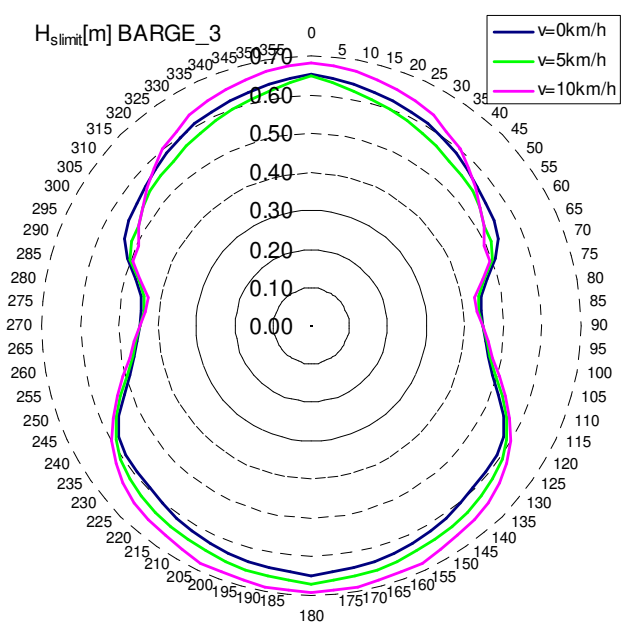

Fig.11.a The operation limits $H_{s}[\mathrm{~m}]$, dynamic criteria, case 3 


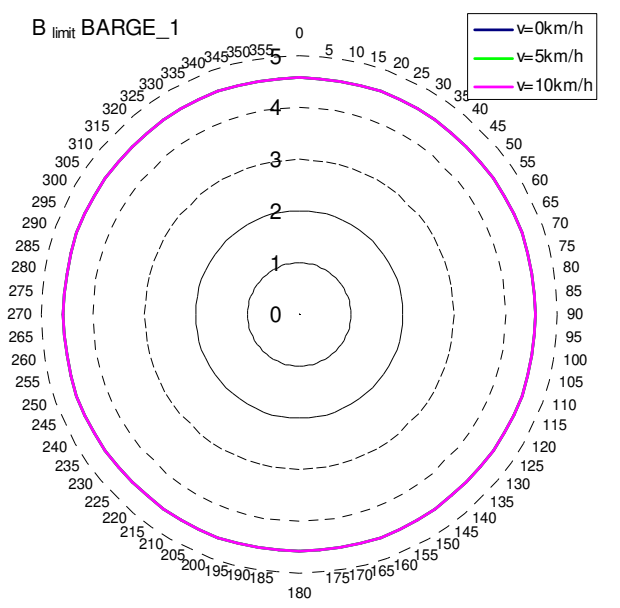

Fig.5.b Beaufort sea state limits, case 3

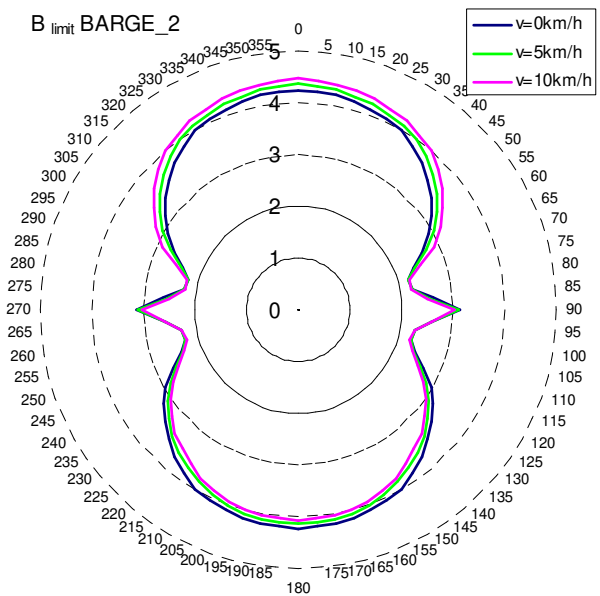

Fig.8.b Beaufort sea state limits, case 2

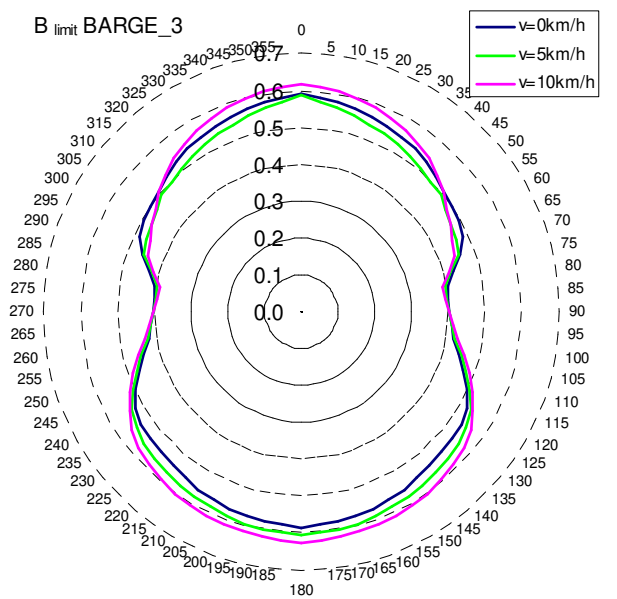

Fig.11.b Beaufort sea state limits, case 3

\section{CONCLUSIONS}

Tables 4.a,b,c include the maximum most probable amplitudes, $R M S$ statistical values, for main motions and accelerations, compared to the dynamic criteria (Table 2).

Table 4.a. Maximum $R M S$ amplitudes, for $H_{s}[\mathrm{~m}]=2 \mathrm{~m}, \mu=0-360 \mathrm{deg} ., v=0 \mathrm{~km} / \mathrm{h}, F_{n}=0$

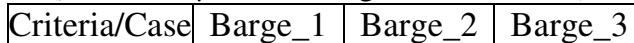

\begin{tabular}{|c|c|c|c|}
\hline$R M S_{z \max }$ aft & 1.483 & 1.500 & 1.611 \\
\hline adm & 2.35 & 0.90 & 0.20 \\
\hline$\%$ & $-36.91 \%$ & $\mathbf{6 6 . 6 2 \%}$ & $>\mathbf{1 0 0 \%}$ \\
\hline$R M S_{z \max }$ mid & 1.263 & 1.228 & 1.412 \\
\hline adm & 2.35 & 0.90 & 0.20 \\
\hline$\%$ & $-46.27 \%$ & $\mathbf{3 6 . 4 2 \%}$ & $>\mathbf{1 0 0 \%}$ \\
\hline$R M S_{z \max }$ fore & 1.567 & 1.537 & 1.629 \\
\hline adm & 3.05 & 1.60 & 0.90 \\
\hline$\%$ & $-48.63 \%$ & $-3.95 \%$ & $\mathbf{8 1 . 0 3 \%}$ \\
\hline$R M S_{\theta \max }$ & 0.0150 & 0.0146 & 0.0143 \\
\hline adm & 0.0349 & 0.0349 & 0.0349 \\
\hline$\%$ & $-57.02 \%$ & $-58.27 \%$ & $-59.13 \%$ \\
\hline$R M S_{\varphi \max }$ & 0.0577 & 0.0510 & 0.0851 \\
\hline adm & 0.0698 & 0.0698 & 0.0698 \\
\hline$\%$ & $-17.31 \%$ & $-27.01 \%$ & $\mathbf{2 1 . 9 0 \%}$ \\
\hline$R M S_{\zeta_{\max }}$ acc & 0.532 & 0.483 & 0.460 \\
\hline adm & 0.9810 & 0.9810 & 0.9810 \\
\hline$\%$ & $-45.77 \%$ & $-50.73 \%$ & $-53.10 \%$ \\
\hline$R M S_{\theta \max }$ acc & 0.018 & 0.016 & 0.015 \\
\hline adm & 0.0256 & 0.0256 & 0.0256 \\
\hline$\%$ & $-30.53 \%$ & $-38.34 \%$ & $-41.48 \%$ \\
\hline$R M S_{\varphi \max }$ acc & 0.134 & 0.072 & 0.151 \\
\hline adm & 0.1790 & 0.1790 & 0.1790 \\
\hline$\%$ & $-25.19 \%$ & $-59.82 \%$ & $-15.81 \%$ \\
\hline $\boldsymbol{H}_{s} / \boldsymbol{B}$ limit & $\mathbf{2 . 0 0 0 / 4 . 5 8 1 . 2 7 8 / 2 . 2 1 0 . 4 4 0 / 0 . 4 0}$ \\
\hline
\end{tabular}

Table 4.b. Maximum $R M S$ amplitudes, for $H_{s}[\mathrm{~m}]=2 \mathrm{~m}, \mu=0-360$ deg., $\nu=5 \mathrm{~km} / \mathrm{h}, F_{n}=0.051$ \begin{tabular}{|c|c|c|c|}
\hline Criteria/Case & Barge_1 & Barge_2 & Barge_3 \\
\hline
\end{tabular}

\begin{tabular}{|c|c|c|c|}
\hline$R M S_{z \max }$ aft & 1.481 & 1.507 & 1.611 \\
\hline $\mathrm{adm}$ & 2.35 & 0.90 & 0.20 \\
\hline$\%$ & $-36.97 \%$ & $\mathbf{6 7 . 4 0 \%}$ & $\mathbf{> 1 0 0 \%}$ \\
\hline$R M S_{z \max }$ mid & 1.262 & 1.228 & 1.411 \\
\hline $\mathrm{adm}$ & 2.35 & 0.90 & 0.20 \\
\hline$\%$ & $-46.28 \%$ & $\mathbf{3 6 . 4 0 \%}$ & $>\mathbf{1 0 0 \%}$ \\
\hline$R M S_{z \text { max }}$ fore & 1.572 & 1.549 & 1.630 \\
\hline adm & 3.05 & 1.60 & 0.90 \\
\hline
\end{tabular}




\begin{tabular}{|c|c|c|c|}
\hline$\%$ & $-48.46 \%$ & $-3.19 \%$ & $\mathbf{8 1 . 1 6 \%}$ \\
\hline$R M S_{\theta \max }$ & 0.0155 & 0.0152 & 0.0149 \\
\hline $\mathrm{adm}$ & 0.0349 & 0.0349 & 0.0349 \\
\hline$\%$ & $-55.47 \%$ & $-56.55 \%$ & $-57.44 \%$ \\
\hline$R M S_{\varphi \max }$ & 0.0577 & 0.0509 & 0.0844 \\
\hline $\mathrm{adm}$ & 0.0698 & 0.0698 & 0.0698 \\
\hline$\%$ & $-17.31 \%$ & $-27.02 \%$ & $\mathbf{2 0 . 8 8 \%}$ \\
\hline$R M S_{\zeta \max }$ acc & 0.531 & 0.483 & 0.461 \\
\hline $\mathrm{adm}$ & 0.9810 & 0.9810 & 0.9810 \\
\hline$\%$ & $-45.85 \%$ & $-50.73 \%$ & $-52.97 \%$ \\
\hline$R M S_{\theta \max }$ acc & 0.020 & 0.018 & 0.017 \\
\hline adm & 0.0256 & 0.0256 & 0.0256 \\
\hline$\%$ & $-21.87 \%$ & $-30.16 \%$ & $-33.75 \%$ \\
\hline$R M S_{\varphi \max }$ acc & 0.134 & 0.072 & 0.148 \\
\hline adm & 0.1790 & 0.1790 & 0.1790 \\
\hline$\%$ & $-25.19 \%$ & $-59.80 \%$ & $-17.27 \%$ \\
\hline $\boldsymbol{H}_{s} / \boldsymbol{B}$ limit & $\mathbf{2 . 0 0 0 / 4 . 5 8} \mathbf{1 . 2 8 0 / 2 . 2 2 0 . 4 3 5 / 0 . 3 9}$ \\
\hline
\end{tabular}

From the dynamic analysis in irregular waves of the Europe B2 1740T barge results: -In the case of light displacement there are no restrictions from the dynamic criteria, so the navigation class is $\mathrm{IN}(2.0)$.

-In the case of common cargo load the restrictions by dynamic criteria occur on vertical motions at aft and amidships, so the navigation class is $\operatorname{IN}(1.2)$.

-In the case of full cargo load the restrictions by dynamic criteria occur on vertical motions at aft, amidships and fore, plus on the roll motion, so the navigation class is $\operatorname{IN}(0.4)$.

If the freeboard safety condition $f_{s}$ is reduced from $0.30 \mathrm{~m}$ to $0.10 \mathrm{~m}$, then in this case the navigation class becomes IN $(0.6)$, that has to be accepted by special approval.

-Because $F_{n \text { max }} \approx 0.1$ the speed influence is reduced on the dynamic responses in the three analyzed cargo loading cases.

Table 4.c. Maximum $R M S$ amplitudes, for

$H_{s}[\mathrm{~m}]=2 \mathrm{~m}, \mu=0-360$ deg. $, v=10 \mathrm{~km} / \mathrm{h}, F_{n}=0.102$

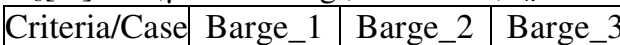

\begin{tabular}{|c|c|c|c|}
\hline$R M S_{z \max }$ aft & 1.478 & 1.521 & 1.643 \\
\hline adm & 2.35 & 0.90 & 0.20 \\
\hline$\%$ & $-37.09 \%$ & $\mathbf{6 8 . 9 7 \%}$ & $>\mathbf{1 0 0 \%}$ \\
\hline$R M S_{z \max } \operatorname{mid}$ & 1.262 & 1.226 & 1.409 \\
\hline $\mathrm{adm}$ & 2.35 & 0.90 & 0.20 \\
\hline$\%$ & $-46.31 \%$ & $\mathbf{3 6 . 2 5 \%}$ & $>\mathbf{1 0 0 \%}$ \\
\hline$R M S_{z \max }$ fore & 1.573 & 1.563 & 1.662 \\
\hline adm & 3.05 & 1.60 & 0.90 \\
\hline$\%$ & $-48.44 \%$ & $-2.33 \%$ & $\mathbf{8 4 . 7 1 \%}$ \\
\hline$R M S_{\theta \max }$ & 0.0159 & 0.0155 & 0.0152 \\
\hline adm & 0.0349 & 0.0349 & 0.0349 \\
\hline$\%$ & $-54.39 \%$ & $-55.55 \%$ & $-56.36 \%$ \\
\hline$R M S_{\varphi \max }$ & 0.0577 & 0.0509 & 0.0843 \\
\hline adm & 0.0698 & 0.0698 & 0.0698 \\
\hline$\%$ & $-17.31 \%$ & $-27.03 \%$ & $\mathbf{2 0 . 7 1 \%}$ \\
\hline$R M S_{\zeta \max }$ acc & 0.540 & 0.493 & 0.466 \\
\hline adm & 0.9810 & 0.9810 & 0.9810 \\
\hline$\%$ & $-44.92 \%$ & $-49.71 \%$ & $-52.49 \%$ \\
\hline$R M S_{\theta \max }$ acc & 0.022 & 0.020 & 0.019 \\
\hline adm & 0.0256 & 0.0256 & 0.0256 \\
\hline$\%$ & $-12.51 \%$ & $-21.60 \%$ & $-25.87 \%$ \\
\hline$R M S_{\varphi \max }$ acc & 0.134 & 0.072 & 0.148 \\
\hline adm & 0.1790 & 0.1790 & 0.1790 \\
\hline$\%$ & $-25.19 \%$ & $-59.78 \%$ & $-17.41 \%$ \\
\hline $\boldsymbol{H}_{s} / \boldsymbol{B}$ limit & $\mathbf{2 . 0 0 / 4 . 5 8}$ & $\mathbf{1 . 2 7 6 / 2 . 2 0 0 . 4 3 1 / 0 . 3 9}$ \\
\hline
\end{tabular}

\section{Acknowledgements}

In this study the program codes package were developed in the frame of Research Centre of the Naval Architecture Faculty from "Dunarea de Jos" University of Galati.

We express our thanks to NAVROM S.A. Galati for granting us the data for the barge type B1740T Europe 2B [4].

\section{REFERENCES}

[1]. Bertram, V, "Practical ship hydrodynamics", Butterworth Heinemann, Oxford, 2000.

[2]. BV,"Rules for the classification of steel ships", Bureau Veritas, Paris, 2017.

[3]. Bidoae, R., Ionas, O.,"Ship theory", E.D.P. Publishing House, Bucharest, 2004.

[4]. Pacuraru, F., Domnisoru, L., Popescu, G., Burlacu, E., "Comparative analysis of the barge type B1740t Europe B2 numerical models on processing the draught survey da$t a$ ", The Annals of "Dunarea de Jos" University of Galati, Fascicle XI Shipbuilding, pp.19-24, 2016.

[5]. Domnisoru, L.,"Ship dynamics. Oscillations and vibrations", Technical Publishing House, Bucharest 2001.

Paper received on December $20^{\text {th }}, 2018$ 